\title{
Toward a Complete Agent-Based Model of a Honeybee Colony
}

\author{
Jérémy Rivière ${ }^{1}$, Cédric Alaux ${ }^{2}$, Yves Le Conte ${ }^{2}$, Yves Layec ${ }^{3}$ \\ André Lozac'h ${ }^{3}$, Vincent Rodin ${ }^{1}$, Frank Singhoff ${ }^{1}$ * \\ 1 Lab-STICC, Université de Bretagne Occidentale, CNRS UMR 6285, Brest, France \\ \{first name.surname\}@univ-brest.fr \\ 2 INRA, UR 406 Abeilles et Environnement, Avignon, France \\ 3 GDSA29, Finistère, France
}

\begin{abstract}
The agent-based approach has been successfully used in the past years to model and simulate complex systems. We use this approach on a honeybee colony in a Dadant hive, where several tens of thousands of bees interact, in order to evaluate the impact of local actions at the bee-level (such as beekeeping practices) on the global system. In this article, we focus on the foraging activity, its recruitment mechanisms and the behaviour of foraging bees, and how these bees interact with the hive's environment, greatly different in scale. We present a customizable, agent-compliant module called the Ecosystem Module, that aims at modelling and simulating the foraging, according to the local weather and the surrounding nectar sources. First results back up our model, showing that these recruitment mechanisms lead to a self-organizing process of the best available sources' selection by the agents.
\end{abstract}

\section{Introduction}

Complex systems are commonly referred to "systems in which large networks of components with no central control and simple rules of operation give rise to complex collective behaviour" [10]. The agent-based approach, because it focuses on the individual to obtain the overall behaviour by emergence, is particularly suited to model and simulate complex systems $(e . g .[8,3]$ in the past few years).

A honeybee colony, where several tens of thousands of honeybees interact and live together, is a great example of complex system. Indeed, one of the notable characteristics of Apis mellifera L., used in beekeeping, and Apis bee species, is that they live in colonies based on eusocial behaviour patterns [20]. This social organisation depends on the multiple interactions (e.g. pheromones [18], nutrition, communication) occurring between specialized individuals (forager, nurse, builder, etc.) of different casts and ages. From these interactions, complex phenomena thus emerge at the system-level (the colony) allowing its self-regulation and self-adaptation, including but not limited to:

* The final authenticated publication
https://doi.org/10.1007/978-3-319-94779-2_42


- Changing roles depending on the needs of the colony [5];

- Thermoregulation and ventilation [17] and formation of the brood nest [6];

- Selection of the best available nectar sources [16, 7].

Our undertaking is to model these phenomena to simulate a honeybee colony. The objective is twofold. First, we aim at allowing direct interactions with bees via a 3D simulation, for two different uses: an educational use (e.g. to learn good beekeeping practices), and a scientific use, to evaluate ecological phenomena (parasites, virus) and anthropogenic factors. Second, investigating these phenomena may lead to identify the core mechanisms behind them, which, from an engineer's point of view, could help to resolve similar issues in other fields. For example, the bees' ability to detect high profitable sources and quickly exploit them before they fade is very efficient, and could inspire new organisation methods in (critic) resource allocation problems.

Some of these phenomena may be described at the colony-level by using differential equations and by not considering bees as individuals $[1,12,11]$. However, in order to investigate these phenomena and to allow interactions with bees, a bottom-up approach is required to deduce the effects of these micro actions in the short and long run on the colony. Discovering, formalizing and modelling the links between individuals (micro-level) and the behaviour of the system (macrolevel) remain a major issue in complex systems. Population models, because they focus on the macro-level, do not take into account the micro-level and its existing links with the macro [4]. We thus choose to model the honeybee colony following an agent-based approach, where the honeybee is an agent, described by its biological cycle, behaviour, and its interactions with its environment.

We present in this article an agent-based model of foraging bees, and how their interactions and recruitment lead to the selection of the best nectar sources in the surroundings of the colony. Focusing on the hive to allow local actions, such as the removal of a frame, a beekeepers practice, makes the hive the obvious agents' environment, with the need to define a high-resolution behaviour for the bees within the hive. However, foraging bees go outside the hive to bring back resources (nectar, pollen, water), within a range of approximately $10 \mathrm{~km}$ [14], for an area of $314 \mathrm{~km}^{2}$. There is thus great differences in granularity and scale between the hive (usually $38 \times 45 \times 31 \mathrm{~cm}$ ) and the outside environment, making the connection between the two environments difficult.

We tackle this issue by designing an Ecosystem Module. This customizable module is compliant with an agent-based simulation: Forager Agents, described in section 4 , interact with this module which rests upon the local weather, the days' length, and the surrounding pollen and nectar sources (see section 3) to simulate the outside trip and bring back resources according to probabilities deduced, adapted or borrowed from previous models in literature.

In the next section, we take a look at some important related works on agentbased simulations of honeybee foraging and recruitment. Preliminary results of experiments conducted under the conditions (weather, sources) of the last three years in Brest (Bretagne, France) with our agent based-model and the Ecosystem 
Module are presented and discussed in section 5. Finally, we conclude and outline the perspectives of this work in section 6 .

\section{Agent-Based Models of Foraging and Recruitment}

As stated by previous works $[13,15]$, recruitment is the cornerstone of a selforganization process that leads foraging bees to select the more profitable sources in the hive's surroundings. Among foraging bees, the scouts are basically explorers, going outside the hive to discover nectar and pollen sources, forage, and communicate their location and profitability (the waggle dance) to unemployed bees, waiting for to be recruited, when coming back at the hive. Surprisingly, the scouts represent a small proportion of the foraging force (around 10\% [14]); and they can only convey the information to a small number of bees, recruiting 0.8 bees on average at each dance [7]. Seeley [14] also noticed that the colony's resources stock leads foraging bees to scout more often and to accept more easily a less profitable source when it is low. It is still unclear however how the stock's state is assessed by the bees, although local perceptions may play a role.

Most of agent-based models focusing on foraging are independent models built to investigate precise biological questions [7, 19]. For example, Dornhaus et al. [7] conducted an experiment based on their model, aiming at quantifying the benefits of recruitment according to the size of the colony and the sources quality. This model introduces some interesting probabilities, adapted in our model, and a first line of approach for the forager agent's behaviour. However, in most cases, these models can hardly be integrated with a model of the colony in the hive because of the difference between the models' time and spatial scales.

On the other hand, a few agent-based models interact with other models in order to simulate the entire colony. In Beehave [1], the agent-based model, dedicated to the foraging activity, interacts with two other population-based modules simulating the life of the colony and the propagation of the parasite Varroa destructor in the colony. The agent-based model includes a spatially explicit landscape that the forager agents explore and on which they forage at flower patches. In this module, foragers are super-individuals representing 100 identical foragers. The model is executed once per day, and the time step of the simulation seems to be one minute. These last two parameters make difficult its adaptation to an agent-based model of the colony in the hive.

To conclude this section, we can draw one common characteristic from all these models: they mostly agree on the nature of the hive's environment, made up of nectar and pollen sources and the weather conditions.

\section{The Ecosystem Module}

The Ecosystem Module aims at simulating the foraging, at an agent's request, according to the outside environment of the hive. As seen in section 2, two important factors are needed to take into account and evaluate the ecosystem's impact on the colony: a representation of the local climate, and the available 
pollen and nectar sources at the current time of the year. These two factors are each the target of a dedicated customizable module, the Weather and the Sources Modules.

The trip conditions of foraging bees are strongly influenced [14] by (1) the day's length: bees can forage only during daylight hours; and (2) the weather: foraging bees can go outside the hive only when the temperature is above approximately $11^{\circ} \mathrm{C}$, and when there is neither rain nor strong wind.

The Weather Module tackles these aspects by, firstly, approximating the time of sunrise and sunset, and secondly, providing real weather data. These aspects are customizable according to the current date and location. The weather data come from the Open Data service (Synop) of the French national meteorological service, Meteo France ${ }^{4}$. The Synop data are weather records for sixty-two stations in metropolitan France and Overseas territories of France, providing the current temperature, humidity, cloudiness etc. From these records and the time of the day, the Weather Module gives information on whether or not a foraging bee is able to go outside the hive.

The Sources Module allows to represent the surrounding nectar and pollen resources, their distance to the hive, their flowering duration and peak, and the sugar concentration of the available nectar, if present. When running a simulation, one can choose to generate a given number of random sources, and can also configure the main local sources. For example, some of the known sources around Brest, in France, and used in the experiment (see section 5), are: the rape, that flowers from the 15th of April to the 15th of May, the bramble, from the 15th of June to the 15th of July, but also the sweet chestnut, etc.

Each source is ruled by a source factor, giving the accessibility of the nectar and/or pollen resources according to the date. The source factor (SF) is directly inspired by the season factor of the HoPoMo model [12], and is thus defined by the following normalized Gaussian function, where $s r c$ is the source, $n b$ is the day's number of the year, $d$ is the flowering duration and $p$ its peak:

$$
S F(\operatorname{src}, n b)=1-\left(\frac{1}{\frac{d}{4} \cdot \sqrt{2 \cdot \Pi}} \cdot \exp \left(-\frac{(n b-p)^{2}}{2 \cdot \frac{d^{2}}{4}}\right)\right)
$$

Each source has also a profitability, computed from its distance to the hive, the nectar's sugar concentration and the quantity of accessible resources. As stated in [15], this profitability will impact the recruitment of bees, according to their perception of the colony's resources stocks (see section 4.1).

The final component of the Ecosystem Module is the Outside Agent, which is the system's interface. We chose to design this interface as an agent to allow our agent-based model to communicate with the Ecosystem Module. The communication protocol lies on the FIPA-ACL standards: a Forager Agent first sends a request to the Outside Agent to forage at an aimed (ever by recruitment or from its memory) source or at a source it must first discover. According to the

$4 *$ https://donneespubliques.meteofrance.fr/ 
Weather and Sources Modules and the $p_{-}$death and $p_{-}$full_return probabilities (see Table 2), the Outside Agent then either refuses to answer the request (because the Forager Agent died, or it has found no source), or accepts. In this latter case, the Outside Agent communicates (1) the pollen and/or nectar quantity the Forager Agent can bring back to the hive, (2) the nectar quality of the source and (3), the travel time, in number of time steps of the simulation (see section 4.4), the Forager Agent has to wait for to simulate its trip.

\section{The Agent-Based Model of Foraging Bees}

As we focus here on the foraging activity, we only consider the foragers, putting the other bees and the interactions with them aside, to be included in the future. From literature and data gathered by the biologist partners, we thus model the biological cycle of a Forager Agent, its behaviour, and the interactions with its environment. These inputs have also been used to verify and calibrate the model.

\subsection{Recruitment and Self-Organization Mechanisms}

Recruitment and profitability are the prime notions on which an efficient source selection lays (as seen in section 2). To reproduce this self-organizing process, we model the recruitment thanks to the following mechanisms:

- When coming back at the hive, the agent may communicate (dance) to unemployed agents the profitability and the location of the source they have found, according to:

- this profitability value;

- and their perception of the current state of the resources stock.

- The state of resources stock in the hive also weights the agent's decision to follow a recruiter or not, and also the probabilities to go scouting ( $\left.p_{-} s c o u t\right)$ and to go on the dance floor ( $p_{-}$dancefloor).

As presented in the section 5, the preliminary results show that these mechanisms seem sufficient to reproduce this self-organization in our model.

\subsection{Environment}

The Forager Agent's environment consists of the hive, the other agents and the Outside Agent (see Table 1). We chose here a minimalist representation of the hive, including only the values of the pollen and the nectar stock. We state that this representation is currently sufficient to reproduce the self-organizing process in the foraging activity, but, as said in the introduction, our objective includes a complete spatial representation of the hive.

This choice of conception has two consequences. First, the consumption of resources by the bees is simplified and simulated according to the number of bees (foragers, but also larva and other bees) in the hive. Secondly, in a real hive, the perception by the bees of the resources stock's state is a complex 
Table 1. Interaction table of the Forager Agent with its environment.

\begin{tabular}{l||l|l|l}
\hline Interactions & Forager Agent & Hive & Outside Agent \\
\hline \hline Forager Agent & Recruitment & Drop resources & $\begin{array}{c}\text { Send a request } \\
\text { to go outside }\end{array}$ \\
\hline Hive & Stock perception & $\begin{array}{c}\text { Pollen and Nectar } \\
\text { consumption }\end{array}$ & - \\
\hline Outside Agent & $\begin{array}{c}\text { Accept / Refuse } \\
\text { the request to go outside }\end{array}$ & - & - \\
\hline
\end{tabular}

phenomena, only partially understood. In this model, this perception is thus simplified, computed by each bee from the stock quantities, with a random bias to simulate its imprecision.

\subsection{Forager Agent's Behaviour}

Because of its simple graphical representation, the state chart presented in Fig. 1 has been the medium to enable an interdisciplinary discussion between biologists, beekeepers and computer scientists, in order to define the Forager Agent's behaviour. In addition to the interaction table and the table of probabilities and parameters (Table 2), it shows a dynamic view of the agent's behaviour.

In Fig. 1, transitions between states are probabilities and/or conditions verified at each time step by the agent. The Table 2 gives the values of the probabilities used by the Outside Agent ( $p_{-}$full_return and $p_{-}$death) and by the Forager Agent. It also shows the values of the Forager Agent's parameters, which may have a random deviation, in order to introduce variability between the agents.

The Forager Agent thus follows the behaviour defined by this state chart, made of seven states. The most important states are:

- OnTheDanceFloor: this state is the crossroad of many transitions. The agent can come from the Resting state, in order to either wait for to be recruited by another agent or go outside as a scout ( $p_{-}$scout probability). The agent can also come from outside, bringing back resources or not (FullReturn and EmptyReturn states).

- WaitingForToBeRecruited: the agent is ready to receive a message from a recruiting agent (Recruitment state). In this case, it assesses the profitability of the conveyed source according to its biased perception of the colony's resources stock. If the source seems profitable enough, it will try to go outside to forage at this aimed source. In other cases, and if the agent has waited too long, it may also go scouting according to the $p_{-}$scout probability.

- TripOutside: this state simulates the agent's trip outside the hive to bring back resources. It is in this state that it communicates with the Outside Agent and interacts with the Ecosystem Module.

- Recruitment: the agent, after a successful trip outside, goes back to the dance floor and may try to recruit (dance) other agents in the WaitingForToBeRecruited state according to the profitability of the source and its perception 


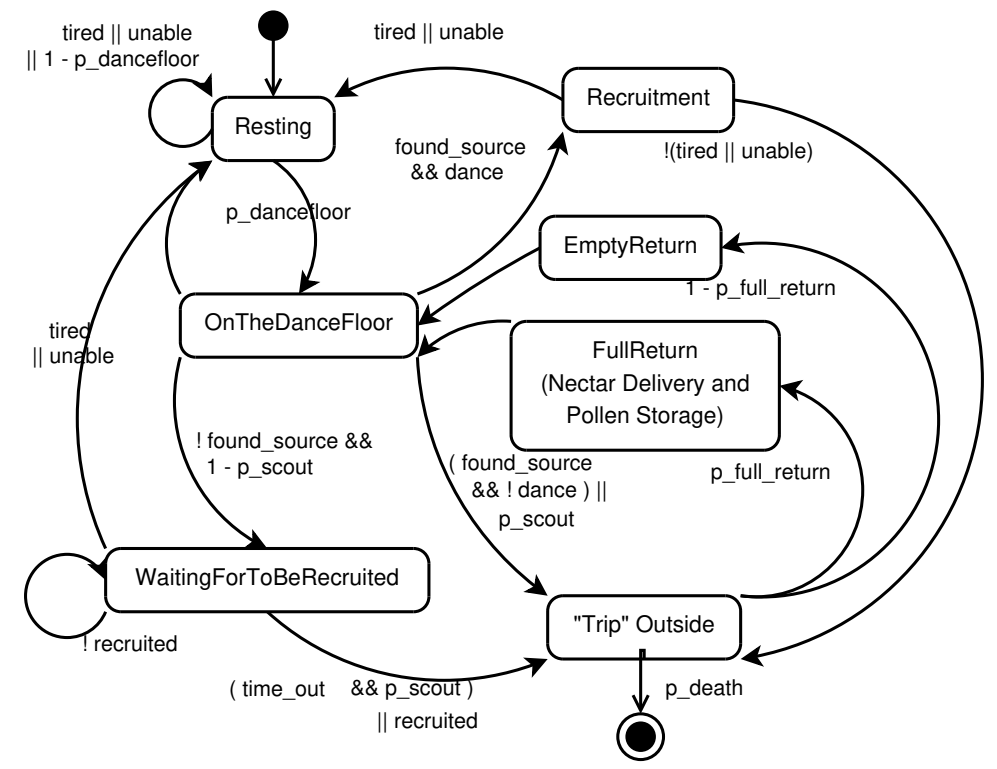

Fig. 1. State chart of the Forager Agent behaviour.

of the colony's resources stock. A source may be profitable (found_source) but not enough regarding the stocks. When recruiting, it sends a message to a nbr_bees_listening with the name of the source and its profitability, then tries to go back outside to the same source if possible.

\subsection{Implementation and Scheduling}

The agent-based model and the Ecosystem Module have been implemented with JADE [2] in Java. JADE provides communication mechanisms and a well-suited architecture for our model: behaviours are independent of agents, and can be added or removed following the evolution of the agent's speciality. JADE also offers a FSMBehavior that allows to directly implement the state diagram and its transitions. Finally, this choice of platform also lies on the necessity to implement the model in a standard programming language to maximise code reliability and make it more easily extendible.

Bees being asynchronous entities, as all living beings, we selected a chaotic asynchronous scheduling, in which the agents are activated in a random manner to avoid a bias in the simulation [9]. As we deal with agents, though, this activation consists in receiving a message from a Scheduler Agent. However, nothing prevents the environment's scheduler (in our case, the Java Virtual Machine) to favour some agents to make more actions than others. The agents thus have to synchronize themselves at some point or another. This is done by introducing the following policy in the agents' behaviour: 
Table 2. Probabilities and parameters used by the Outside and the Forager Agents . Each value is given according to the ratio $\frac{\text { simulation time step }}{\text { real time }}=\frac{1}{1000}$. Probabilities that can be weighted by the colony's resources stock are represented with a *.

\begin{tabular}{|c|c|c|}
\hline Probability/Parameters & Value & Adapted from \\
\hline \multicolumn{3}{|l|}{ Outside Agent } \\
\hline$p$ death & 0.0108 & {$[11]$} \\
\hline \multirow{2}{*}{$p_{-}$full_return } & 0.43 when scouting & {$[16]$} \\
\hline & 0.93 when recruited & {$[16]$} \\
\hline Foraging duration & {$[5.41 ; 11.37] \mathrm{min}$. for $650 \mathrm{~m}$} & {$[15]$} \\
\hline \multicolumn{3}{|l|}{ Max. quantity in one trip: } \\
\hline - of nectar & {$[14.6 ; 16] \mathrm{mg}$} & {$[7]$} \\
\hline - of pollen & $25 m g$ & {$[7]$} \\
\hline Flight speed & $1.29 m . s^{-1}$ & {$[15]$} \\
\hline Nectar consumption in flight & $0.0083 \mathrm{mg} \cdot \mathrm{m}^{-1}$ & {$[14]$} \\
\hline \multicolumn{3}{|l|}{$\overline{\overline{\text { Forager Agent }}}$} \\
\hline$p$ scout $*$ & 0.00825 & {$[7]$} \\
\hline$p_{-}$dancefloor $*$ & 0.001 & Deduced from calibration \\
\hline max_outing_on_a_row & {$[20 ; 30]$ trips in a row. } & {$[14]$} \\
\hline min_go_out_temp & $11^{\circ} \mathrm{C}_{-}^{+} 1$ & {$[14]$} \\
\hline max_go_out_temp & $40^{\circ} \mathrm{C}_{-}^{+} 1$ & {$[14]$} \\
\hline nbr_bees_listening & {$[0 ; 3]$ agents per dance } & {$[7]$} \\
\hline wait_recruit_time & $30 \mathrm{~min}$. & Deduced from calibration \\
\hline
\end{tabular}

1. The Forager Agent waits for the Scheduler Agent's activation message;

2. After reception, it makes $n$ actions;

3. Then, it informs the Scheduler Agent that it has done, and waits again for an activation message.

For its part, the Scheduler Agent waits for all Forager Agents to be done before sending a new activation message, leading to a synchronization of all the agents. The smaller is the value of $n$, the greater will be the number of messages exchanged. On the other hand, a too great $n$ value can lead to great differences between the number of actions done by the agents. These actions are defined within the states introduced above, as steps that take in reality approximately one second to be performed by the bee.

\section{Experiments and Preliminary Results}

We have conducted 3 experiments with our agent-based model and the Ecosystem Module, each conducted under the conditions (local weather and sources) of the last 3 years in Brest (Bretagne, France). The experiments run from the 1st of March to the 1st of September of each year. Each experiment consists in 10 runs with the same weather and location of sources, and the same initial state: 500 foraging bees, and rather low resources stock. As the agent-based model does not yet include the queen and the larva, the $p_{-}$death probability is set to 0 . 
These first experiments aim at verify our model, by notably verifying the following hypothesis:

- H1: The weather influences the outing of Forager Agents, as stated in [14];

- H2: Agents select the best available sources in the surroundings of the hive;

- H3: Finally, Forager Agents scouting represent an average number of around 10\% of the foraging force [14].
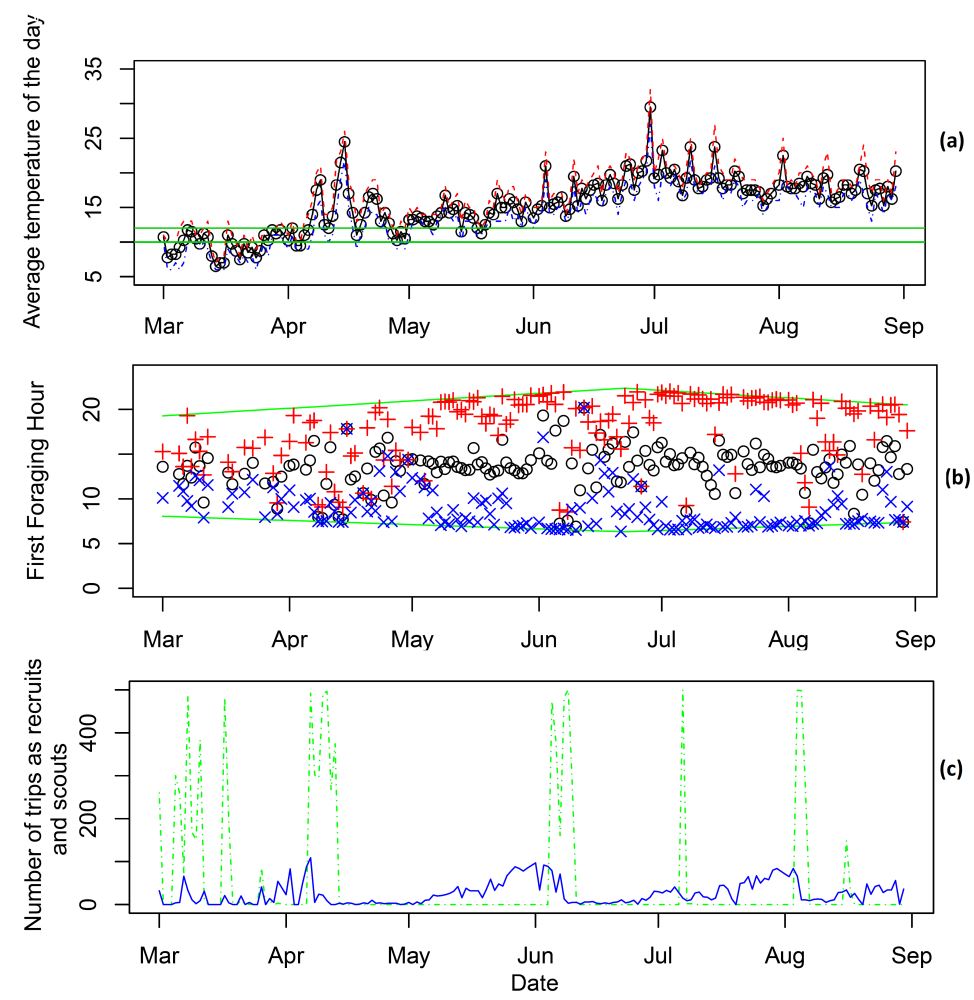

Fig. 2. Graph (a) on the top shows the average temperature per day of 2015 in Brest (circles) and the minimum (dash-dotted line) and maximum (dashed line) averages. Graph (b) shows the average first foraging hour per agent per day (circles), i.e. the time of the day at which the agents go forage for the first time. Minimum $(+)$ and maximum (x) first foraging hour per day are also displayed. Graph (c) shows the number of trips done by scouting agents (solid line) and recruiting agents (dashed line) per day.

\subsection{Preliminary Results}

Fig. 2 first gives an overview of the influence of weather on the outing of Forager Agents (H1). This is one run of the 2015 experiment. The first points on the 
left of the graph (b) mean that there are some agents going out around 10 a.m. $(+)$, while the majority goes outside around 14 p.m. (circle), until 16 p.m. (x). In early March for example, there are some days where the agents cannot go outside at all because of the low temperature or the bad weather. Other days, the average first foraging hour can be very early in the day (e.g. in June).

As we take a look at the recruitment mechanism, graph (c) shows a correlation between the number of Forager Agents scouting outside (solid line) and the recruited agents (dashed line). In early April for example, a new profitable source is discovered by scouts, and, as the weather allows the outing, they recruit a great foraging force toward a source: almost $50 \%$ of the total foraging force. This phenomena is explained by the colony's stock low level, that leads the agents to go more easily on the dance floor and to accept less profitable sources. The number of recruits thus rises as only a few scouts remain. These results are found across all runs of the three experiments.

Based on the results over the three experiments, the average proportion of scouts against the total number of Forager Agents (H3) is between 6\% and $7 \%$. As the stock is low, we expected to find a greater value $(10 \%)$, so more experiments are needed to investigate this point.

Fig. 3 shows the same particular run than the previous figure, to illustrate how the agents select the best available source. Graph (a) shows that, from the 60 th to the 80th days, the source S1 (solid line) is the most profitable. Then, a second source S2 (dash-dotted line), shorter in duration, becomes the best source for approximately 10 days. Finally, after a quick return of S1, a third source S3 (dashed line) takes over from the 95th day.

In the beginning of the run, the low temperature and the bad weather do not allow the agents to efficiently go forage, as we can see in graphs (b) and (c). Three points of interest are then to be noticed. The first one is around the 75th day (mid-March): scouting agents discover sources S1 and S2, and the recruitment leads the agents to leave S2 for S1 after 3 days. The second interesting point is about source $\mathrm{S} 2$, from the 81 th to the 98 th day, ignored by agents, whereas it is the best available source at this moment. This can partly be explained by the fact that scouting agents missed this source during this duration, maybe caused by a too great concentration of agents on $\mathrm{S} 1$ at this time.

Finally, around the 97th day (early April), we find again the great recruitment described earlier (see Fig. 2). At this point, agents foraging at sources S1 and S2 progressively leave these sources for the third one (S3), as scouting agents discover and communicate about this more profitable source.

\section{Conclusion and Perspectives}

We presented in this article an agent-based model of foraging bees, and the Ecosystem Module in relation with the Forager Agent's behaviour. This latter module aims at connecting two environments of different granularity and scales, in time and space, and allows agents to navigate between them. 
Preliminary results of first experiments verify the influence of the environment on the model, and show that the Forager Agent's behaviour successfully reproduces recruitment, leading to a self-organizing process of the best available sources' selection by the agents. These results are a first step toward the verification of our model, that more experiments are planned to fulfil.

The building of the complete agent-based model is currently in progress and includes the definition of the behaviours of other roles and casts, such as the Queen, the larva, etc. In order to formalize and model more complex phenomena occurring within a colony, a spatially explicit hive and resources stock representation is also a work in progress. Ultimately, more experiments conducted on the enhanced agent-based model and the Ecosystem Module will be needed to validate our model by comparing the results with colonies dynamics data. Finally, allowing a user to interact locally with the bees involves to create a
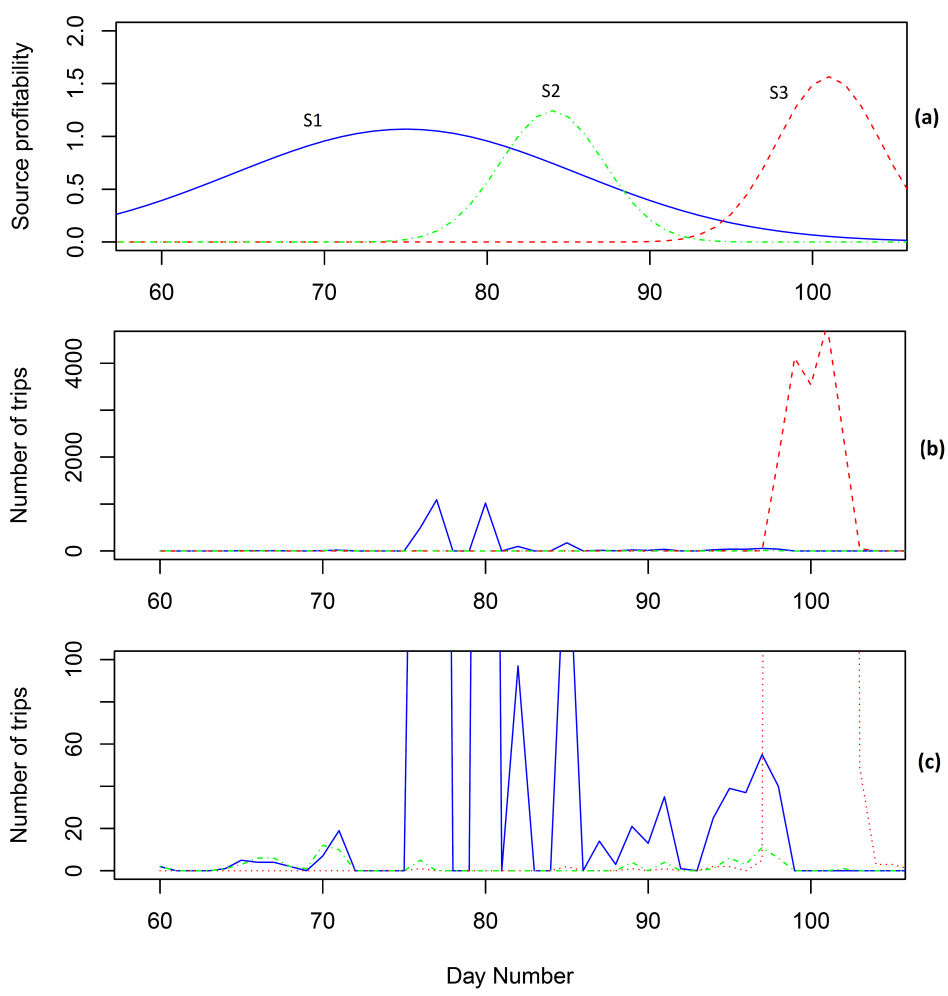

Fig. 3. Graph (a) shows the profitability evolution of three sources between early March and late April 2015. Graph (b) shows the number of trips per day outside the hive according to the same sources displayed on graph (a). The third graph (c) is an enlargement of $(b)$. 
virtual representation of the hive and to integrate visualization and interactions with the colony, which have to be realistic, intuitive and ergonomic.

\section{References}

1. Becher, M.A., Grimm, V., et al.: Beehave: a systems model of honeybee colony dynamics and foraging to explore multifactorial causes of colony failure. Journal of Applied Ecology 51(2), 470-482 (2014)

2. Bellifemine, F., Bergenti, F., Caire, G., Poggi, A.: Jade - a java agent development framework. In: Bordini, R.H., Dastani, M., Dix, J., El Fallah Seghrouchni, A. (eds.) Multi-Agent Programming: Languages, Platforms and Applications, pp. 125-147. Springer US, Boston, MA (2005)

3. Bilbao-Castro, J.R., Barrionuevo, G., et al.: Weaver: A multiagent, spatial-explicit and high-performance framework to study complex ecological networks. In: Bajo, J., Hallenborg, K., et al. (eds.) Highlights of Practical Applications of Agents, Multi-Agent Systems, and Sustainability - The PAAMS Collection. pp. 139-150. Springer (2015)

4. Bonabeau, E.: Agent-based modeling: Methods and techniques for simulating human systems. Proceedings of the National Academy of Sciences 99(suppl 3), 72807287 (2002)

5. Bonabeau, E., Theraulaz, G., Deneubourg, J.L., Aron, S., Camazine, S.: Selforganization in social insects. Trends in Ecology and Evolution 12(5), 188 - 193 (1997)

6. Camazine, S.: Self-organizing pattern formation on the combs of honey bee colonies. Behavioral Ecology and Sociobiology 28(1), 61-76 (Jan 1991)

7. Dornhaus, A., Klügl, F., Oechslein, C., Puppe, F., Chittka, L.: Benefits of recruitment in honey bees: effects of ecology and colony size in an individual-based model. Behavioral Ecology 17(3), 336-344 (2006)

8. Krichene, H., El-Aroui, M.A.: Behavioral and informational agents-based modeling and simulation of emerging stock markets. In: Bajo, J., Hernández, J.Z., , et al. (eds.) Trends in Practical Applications of Agents, Multi-Agent Systems and Sustainability. pp. 3-10. Springer (2015)

9. Kubera, Y., Mathieu, P., Picault, S.: How To Avoid Biases In Reactive Simulations. In: Demazeau, Y., Pavòn, J., Corchado, J.M., Bajo, J. (eds.) Proceedings of the 7th International conference on Practical Applications of Agents and Multi-Agents Systems (PAAMS'2009). Advances in Intelligent and soft computing, vol. 55, pp. 100-109. Springer, Spain (2009)

10. Mitchell, M.: Complexity: A Guided Tour. Oxford University Press (2009)

11. Russell, S., Barron, A.B., Harris, D.: Dynamic modelling of honey bee (apis mellifera) colony growth and failure. Ecological Modelling 265, 158 - 169 (2013)

12. Schmickl, T., Crailsheim, K.: Hopomo: A model of honeybee intracolonial population dynamics and resource management. Ecological Modelling 204(1), 219 - 245 (2007)

13. Seeley, T.D.: Division of labor between scouts and recruits in honeybee foraging. Behavioral Ecology and Sociobiology 12(3), 253-259 (Jun 1983)

14. Seeley, T.D.: The Wisdom of the Hive. Harvard University Press (1995)

15. Seeley, T.D., Camazine, S., Sneyd, J.: Collective decision-making in honey bees: how colonies choose among nectar sources. Behavioral Ecology and Sociobiology 28(4), 277-290 (Apr 1991) 
16. Seeley, T.D., Visscher, P.K.: Assessing the benefits of cooperation in honeybee foraging: search costs, forage quality, and competitive ability. Behavioral Ecology and Sociobiology 22(4), 229-237 (Apr 1988)

17. Sumpter, D.J., Broomhead, D.S.: Shape and dynamics of thermoregulating honey bee clusters. Journal of Theoretical Biology 204(1), 1 - 14 (2000)

18. Traynor, K.S., LeConte, Y., Page, R.E.: Queen and young larval pheromones impact nursing and reproductive physiology of honey bee (apis mellifera) workers. Behavioral Ecology and Sociobiology 68(12), 2059-2073 (2014)

19. de Vries, H., Biesmeijer, J.C.: Modelling collective foraging by means of individual behaviour rules in honey-bees. Behavioral Ecology and Sociobiology 44(2), 109-124 (1998)

20. Winston, M.L.: The Biology of the Honey Bee. Harvard University Press (1987) 